\title{
VISION-BASED HAZARD DETECTION
}

\section{CHIU-HUNG LUK}

Department of Computer and Electrical Engineering, OGI School of Science and Engineering, OHSU, Beaverton, OR 97006, USA

DAN HAMMERSTROM

Department of Electrical and Computer Engineering, Portland State University, Portland OR 97207, USA

\author{
MAZAD S. ZAVERI
}

Department of Electrical and Computer Engineering, Portland

State University, Portland, OR 97207, USA

\section{RICHARD J. KERR}

Max-Viz, Inc., 16165 SW 72 ${ }^{\text {nd }}$ Ave, Portland, OR 97224, USA

\begin{abstract}
Advances in sensor and GPS technologies make possible better guidance tools for vehicle/aerial navigation. In the work reported here we look at the detection of hazards, such as aircraft or other objects, on or near the target runway. Our system consists of two modules: 1) regions of interest (ROI) detection, and 2) hazard recognition. One of the harder problems in object recognition is to segment the target object from a cluttered background. In this system we use a "poor man's" segmentation, by taking advantage of the fact that we have an approximate reference that we can differentiate from. The regions of interest are defined as significant differences between the input image and the reference image. Since this differencing can be complex due to the fact that the images may not be precisely registered, we employed a novel histogram method which is reasonably invariant to spatial transformations for ROI detection.
\end{abstract}

\section{INTRODUCTION}

Advances in sensor and GPS technologies have opened up the opportunity of developing better guidance tools for aerial/ground navigation. These guidance tools integrate data routinely from different sensors and GPS and then present to the pilot integrated information about the environment for safe navigation and landing. One important part of the guidance tools is hazard/obstacle detection. As the name implies, it searches throughout the scene to detect if there is any possible hazard (aircraft or truck, for example) on or near the runway and determines the type of hazard if present. Our research goal is to develop such hazard detection using a biologically inspired approach.

Existing vision-based methods for obstacle detection can be classified into two types: feature based and optical flow based. In feature based methods (Sridhar B. et al., 1993), significant features are detected from the images and matching between features are made from frame to frame using normalized correlation. But since only features are used, the choice of the feature set becomes very critical for the success of the method. Also any transformation of the features due to scaling or rotation would deteriorate its performance.

Optical flow methods based on estimation of temporal or spatial disparities from the image sequences have also been suggested (Sull S. and Sridhar B., 1995). A global flow field is first computed from the whole image. A threshold 
for the temporal/spatial disparity of the obstacle is then manually set and any part with estimated disparity higher than threshold is considered as an obstacle or a portion of an obstacle. As can be expected, obstacle detection is affected by the value of this threshold and the successful discrimination between observed disparity due to obstacles and observed disparity due to sensor noise.

So far, vision based hazard detection is still a difficult problem, with few general purpose solutions. Here we propose a detection method that is similar to the first approach. But instead of just using features, we used the whole image for obstacle detection. Also, we employed histogram correlation rather than normalized correlation method in the matching process.

\section{METHOD}

Figure 1 shows the schematic diagram of our hazard detection system. It consists of two modules: a region of interest (ROI) detection unit and an ROI classification unit. The inputs to the system are two images: an incoming image obtained directly from sensor at the time, and a reference image which is retrieved from an image database. The algorithm of how to retrieve the suitable reference image from the database is beyond the scope of the current paper and interested readers should consult our previous publications (Kerr J. R. et al. 2003, Luk C. H. et al., 2004) for more details.

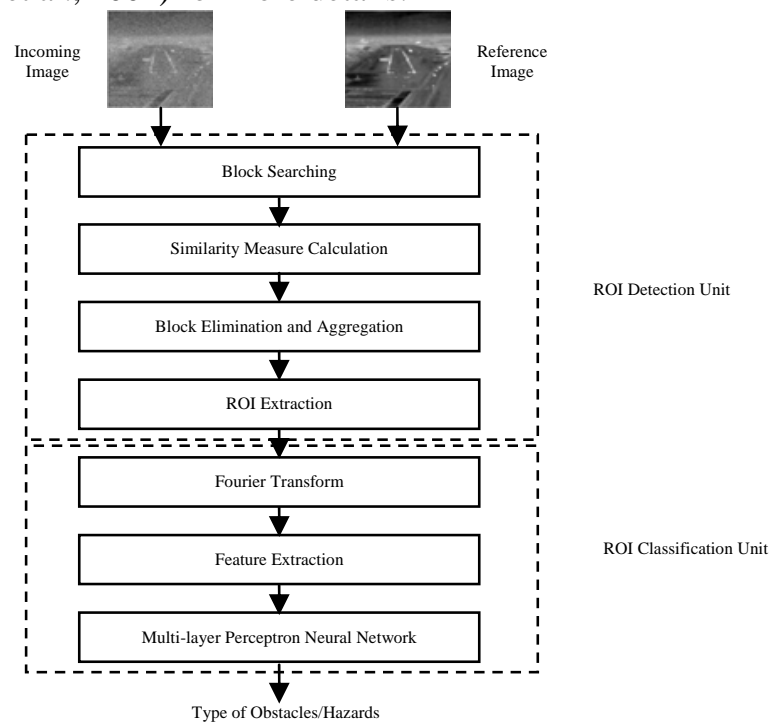

Figure 1: Schematic diagram of hazard detection system

The reference and real images are not perfectly registered due to the limitations of sensors and our system, and are moderately misaligned by translation, rotation and scaling. This introduces difficulty in the detection of obstacles as we need to discriminate differences due to the presence of hazards from differences due to misalignment. The goal of our ROI detection unit is to find out suspicious portions of input image that may contain hazards. ROI 
classification unit is then used to classify the type of obstacles in the segmented regions. The detailed algorithm of each unit is now described.

\section{ROI detection unit}

As shown in Figure 2, it consists of three main steps: block searching, similarity measure calculation, block aggregation and elimination.

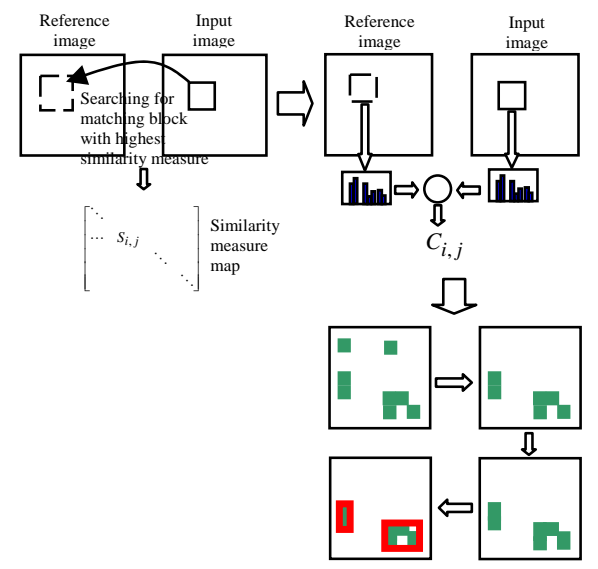

Figure 2: Flow diagram of region of interest (ROI) detection unit

Inputs:

Incoming and reference images are misaligned within \pm 10 pixels in horizontal and vertical directions. The input image is a translated, rotated and slightly scaled version of the reference one.

Block Searching:

The real image is first partitioned into blocks of $n \times n$ size. The value of $n$ can be 8,16 or 32 . After partitioning, the algorithm then searches in the neighborhood of each input block for a matching block from the reference image that gives the highest similarity measure. Since we know that the misalignment is only within \pm 10 pixels, searching is constrained. So after operating throughout all blocks, a similarity measure map (for blocks) is created.

Similarity Measure Calculation:

Since any translation between input and reference images could be resolved by a block search, the only transformations we need to handle are rotation and scaling. One way is to develop intensity histograms of the input and reference blocks. As the intensity histogram is only concerned with the number of pixels having certain intensities, it does not matter whether the scene within the block is rotated or slightly scaled as long as most of the pixels are still contained in the block. Therefore, we define the similarity measure as the normalized correlation of the intensity histograms of the input and reference blocks. Using correlation eliminates the problem due to the absolute intensity difference between blocks, provided that the relative intensities within the blocks are roughly the same. Aggregation and Elimination:

After obtaining the similarity map, we select the first $m$ blocks with lowest similarity measure. We then clean up any isolated blocks and connect the aggregated blocks together. A high similarity measure means that there is a reference block that is very similar to the input one and hence no significant 
change is found in the input block. A low similarity measure suggests that a significant change has been observed in the input block. After cleaning and connecting, rectangular windows are developed to contain the aggregated blocks.

Output:

Input image with only the regions of interest being highlighted.

\section{ROI classification unit}

ROIs are then classified using a Multi-Layer Perceptron Neural Network (MLP-NN (Simon Haykin, 1998). The training data for the MLP-NN is prepared using the following steps (all ROI images go through these steps):

Step 1:

All ROIs are first separated by using connected component labeling according to the Matlab's Image Processing Toolbox User Guide. (Note: Each rectangular ROI is assumed to be unconnected to other ROIs in the image, and each ROI could be of different sizes and different aspect ratios.)

Step 2:

For each separated ROI, a feature vector is generated as follows (Lendaris G. G. and Stanley G. L., 1970):

a) The 2D-Fourier Transform (FT) of the ROI is taken, and the obtained FTspace (2D space in the frequency domain) is divided into $n r$ annular rings as shown in Figure 3 (a). The ring-feature vector of size $[1 \mathrm{x} n r]$ is formed by summing the absolute values of the FT intensities in each ring in the FT space. These ring-features are roughly rotation invariant.

b) The FT-space is divided into $n w$ wedges as shown in Figure 3 (b), and in a similar way as described above (part (a) of Step 2), the wedge-feature vector of size $[1 \times n w]$ is formed. These wedge features are roughly scale invariant, and to a small degree rotation-invariant. (Note: The 2D-FT of a ROI containing an object is independent of the position of the object inside the ROI, so invariance to translation is inherent from FT.)

c) The final feature vector is formed by combining both ring and wedge features, and has size of $[1 \times(n r+n w)]$. (Note: The invariance of the features to scaling, rotation will be reduced because we are considering the ROIs to be of different sizes and different aspect ratios, and so the resulting FT-space of different ROIs will be of different sizes and aspect ratios.)

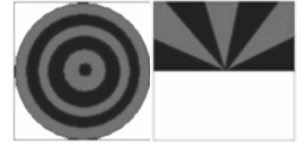

(a)

(b)

Figure 3: FT-space divided into (a) annular-rings and (b) wedges

Step 3:

The feature vector for each ROI, along with its manually assigned classlabel is added to the training data. The training data will thus consist of (featurevector, class-label) pairs for all ROIs separated from all images.

\section{RESULTS AND DISCUSSION}

In this experiment, we used street scenes since we did not have suitable aircraft approach videos which contained hazards. We have taken some street 
photos at different times and slightly different positions to simulate both reference and real images. Figure 4 (a) and (b) show two of the 135 photos taken. We can observe that there are some additional vehicles (defined as obstacles on the street) in the second pictures. There are also some irrelevant changes (changes that should not be classified as obstacles or hazards) - clouds and trees. Moreover, the second image is slightly translated, rotated and scaled from the first one. Figure 4 (c) shows the output of our detection algorithm with the block size set at $16 \times 16$. It can easily be seen that our algorithm intelligently ignores the unimportant changes, such as pedestrians, the wind moving trees, and shadow as the sunshine is intermittent under cloud cover, in the image while keeping the significant changes (new vehicles) in the regions of interest.

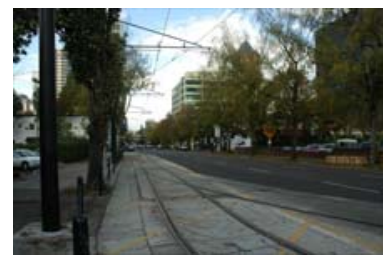

(a)

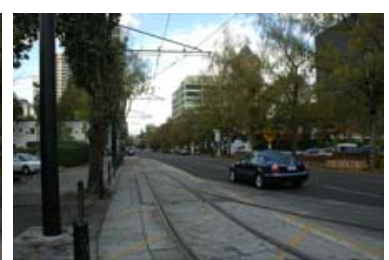

(b)

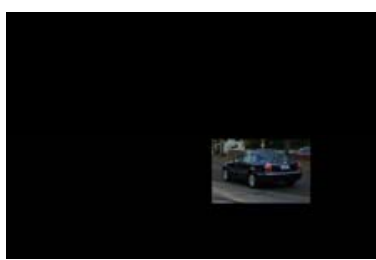

(c)

Figure 4: The inputs (a) and (b) and output of the ROI detection unit

An MLP-NN with 25 inputs, 6 hidden nodes, and 3 output nodes was created. The 25 inputs correspond to 25 features formed by $n r=20$, (we use only first 15 of them) and $n w=10$. The 3 outputs correspond to the 3-bit classlabel (one up code) for the three classes - car, person, and unknown (trees, building, pole, rail-tracks, etc).

The physical data consisted of 132 images of a busy street, each image containing a combination of ROIs - car, person, and unknown. Around 258 different ROIs were obtained from them after hand-eliminating redundant ROIs. The training data was generated from them.

The MLP-NN was trained on randomly selected $60 \%$ of the data, and was tested on $50 \%$ of the data (i.e. remaining $40 \%$ plus randomly selected $10 \%$ ).

The classification accuracy matrix for the experiment is shown below:

\begin{tabular}{|c|c|c|}
\hline Class & Training Data & Test Data \\
\hline Unknown & $94 \%$ & $89 \%$ \\
\hline Car & $98 \%$ & $96 \%$ \\
\hline Person & $100 \%$ & $100 \%$ \\
\hline
\end{tabular}

Table 1: Accuracy rates of different classes using ROI classification unit

For test data, the simple MLP-NN gives a good generalization, i.e. accuracy rates of $100 \%, 96 \%$, and $89 \%$ for person, car, and unknown respectively.

In the current work, we separated hazard detection into two steps: finding ROIs that most possibly contain obstacles, and classifying their types. The use of the histogram correlation method in detecting ROIs is robust and relaxes the requirement for perfect registration between images. Also block searching in ROI detection can be computed in parallel by using FPGA hardware.

The ROI classification based on ring and wedge features yields a good classification as we had expected, based on the following rationale: first, the 
features are roughly invariant to rotation, scaling and translation as shown in Figure 5 (a), and second, the features have sufficient information content in them to perform a satisfactory classification as shown in Figure 5 (b).

This work demonstrates a feasible approach to the hazard detection problem in an aircraft landing guidance system. More work is needed in classifying the types of hazard that may be possible during real aircraft operation.
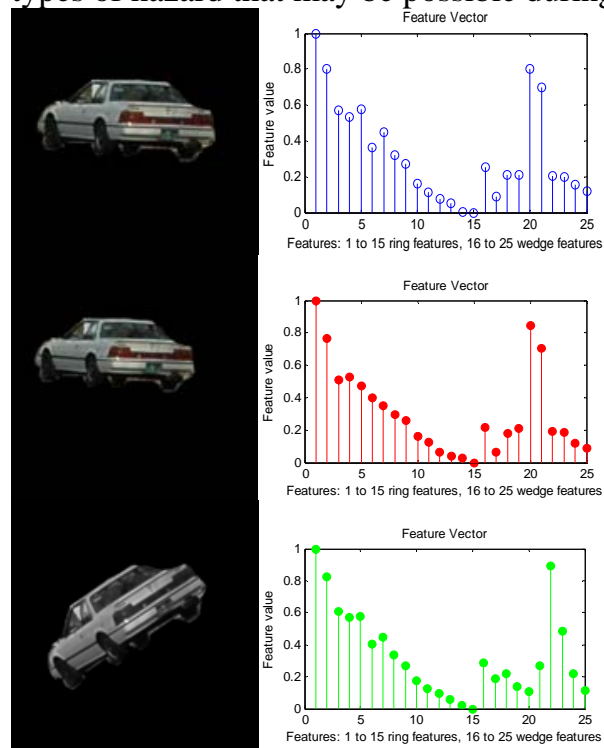

(a)

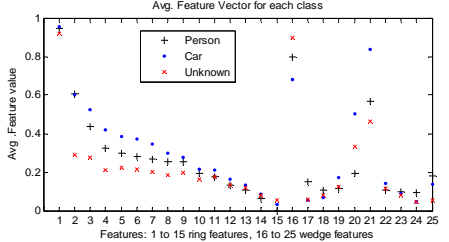

(b)

Figure 5: (a) ROIs and their corresponding feature vectors and (b) average feature vectors for all three classes

\section{ACKNOWLEDGMENTS}

Supported in part by AFRL/SNHC, Hanscom AFB, MA, Contract No. F19628-02-C-0080

\section{REFERENCES}

Kerr, J.R., Luk, C.H., Hammerstrom, D. and Pavel, M., 2003, "Neural Net Based Processor for Robust, High-integrity Multisensor and Synthetic Vision Fusion”, Proceedings, AIAA/IEEE Digital Avionics Systems Conference, Vol. 2, pp. 9.D.4 - 91 - 12

Haykin S., Neural Networks: A Comprehensive Foundation, $2^{\text {nd }}$ Edition, Pearson Education, Inc., 1998

Lendaris G. G., Stanley G. L., 1970, "Diffraction-Pattern Sampling for Automatic Pattern Recognition”, Proceedings of the IEEE, Vol. 58, pp. 198 - 216

Luk C.H., Gao C.J., Hammerstrom D., Pavel M. and Kerr J.R., 2004, "Biologically Inspired Enhanced Vision System (EVS) for Aircraft Landing Guidance”, Proceedings, International Joint Conference on Neural Networks, Vol. 3, pp. 1751 - 1756

Sridhar B., Smith P., Suorsa R. and Hussien B., 1993, "Multirate and Event-Driven Kalman Filters for Helicopter Flight”, IEEE Control Systems, Vol. 13, pp. 26 - 33

Sull S. and Sridhar B., 1995, "Model-Based Obstacle Detection from Image Sequences", Proceedings, International Conference on Image Processing, Vol. 2, pp. 647 - 650 